\title{
Inverse Design of Wind Turbine Blades Using a Vortex Line Method
}

\author{
Seongkyu Lee* \\ Aerodynamics and Acoustics Lab \\ General Electric Global Research Center \\ One Research Circle, Niskayuna, NY, U.S.A. 12309
}

\begin{abstract}
This paper discusses the theory of the VLM code and an inverse design process with the VLM.
\end{abstract}

A flow chart of vortex line method (VLM) is shown in Fig. 1 [1-4]. At start, the blade geometry information including the chord, twist angle, dihedra angle, sweep angel distributions is read along with the operation conditions (TSR and Pitch angle). The rotor power coefficient is estimated based on the operation condition. The pitch of vortex sheet is determined by this rotor power coefficient, and the initial vortex strength is guessed. At the quarter of the chord of each radial station on the blade, the induced velocities are computed using the Biot-Savart Law. An effective angle of attack is computed by the flow angle and the blade twist angle. The lift and drag coefficients are found from a table look-up of the polar data. The polar data implicitly take into account the airfoil blade thickness and flow separation. This polar data can be obtained from measured data or CFD analysis for each airfoil. Once the lift coefficient is found, the circulation is calculated from the Kutta-Joukowski lift theorem. The current VLM code added artificial viscosity to the Kuttal-Joukowski lift theorem in order to provide smooth results at high angle of attach where flow separation occurs. A new circulation distribution changes the induced velocities or flow angle at the blade stations again. This process is iterated until the circulation distribution is converged, which constructs an inner iteration loop. During this process, the wake structure is assumed to be fixed. Once the circulation is converged, the rotor power coefficient is recalculated. This newly updated power coefficient changes vortex structures again, which constructs an outer iteration loop. The outer loop is iterated until the power coefficient is converged. When the power coefficient is converged, the results are printed out and the program is stopped. 


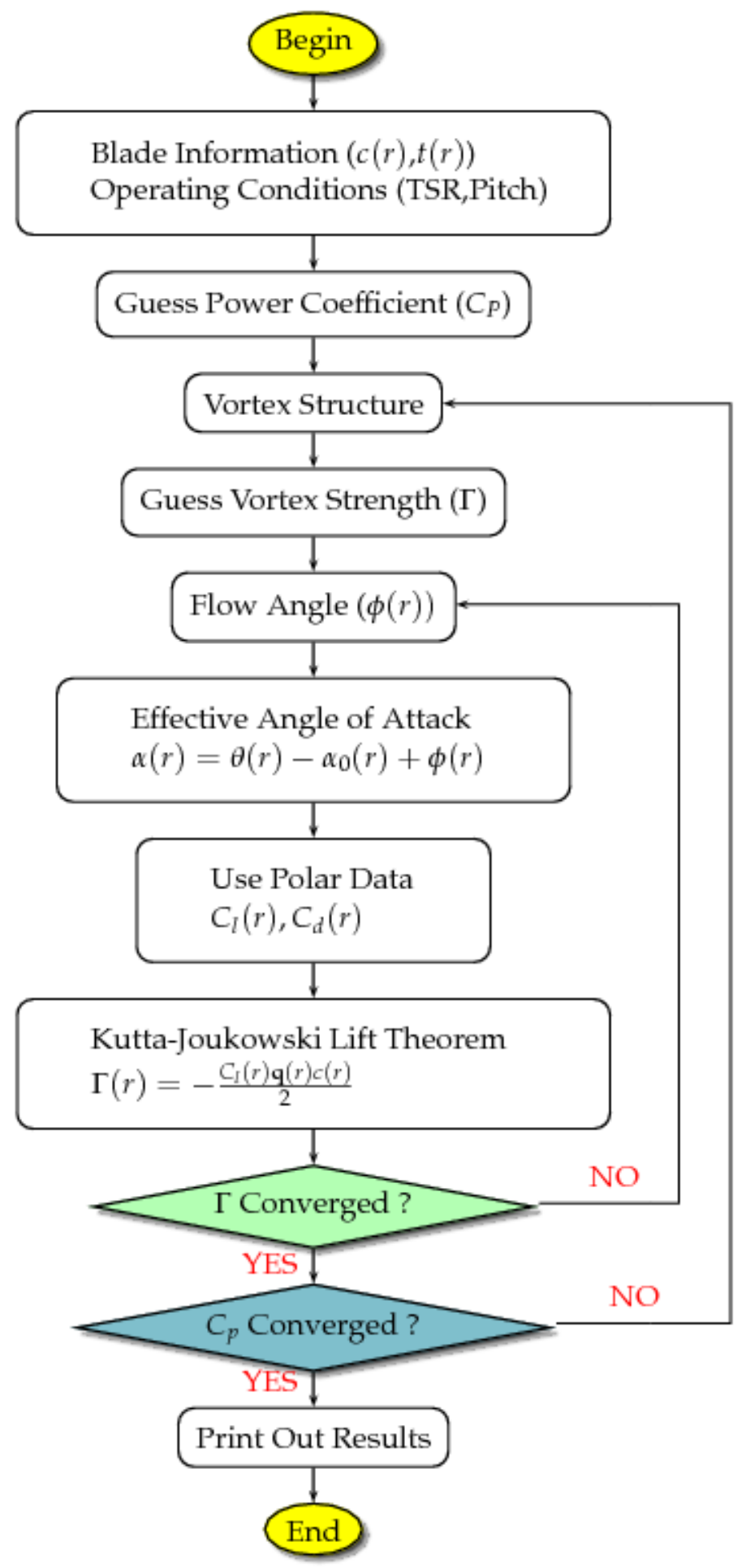

Figure 1: Flow chart of the VLM code. 


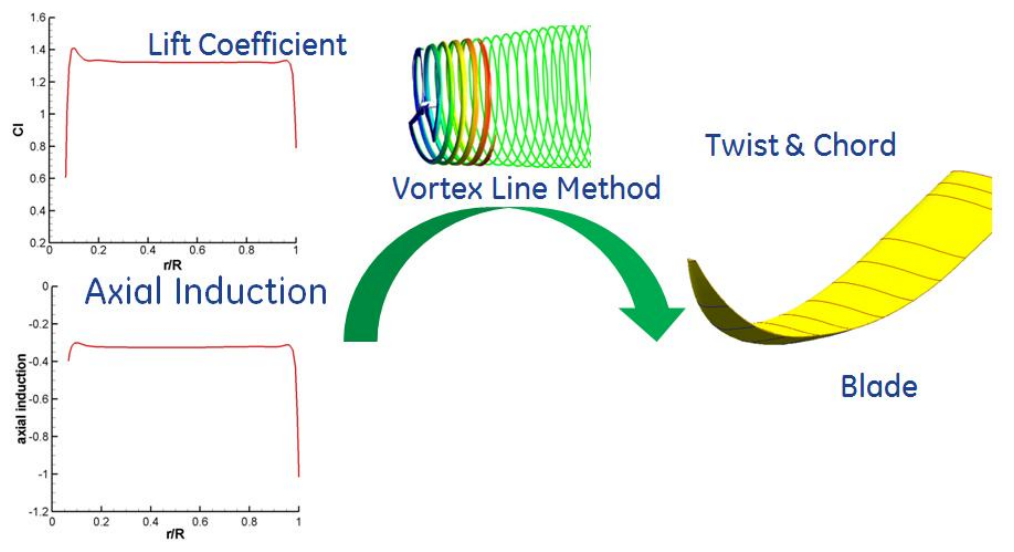

Figure 2: Schematic of the inverse design of wind turbine blades with a vortex line method.

\section{Inverse Design with Vortex Line Method}

An inverse design with the VLM code is described in this section. The purpose of the inverse design is to determine the chord and twist angle distributions based on the desired lift coefficient and axial induction distributions. In the present paper, the multi-dimensional Newton iteration method is used to find the chord and twist angle distributions. In the multi-dimensional Newton method, the unknowns are iteratively found as follows:

$$
\mathbf{x}^{k+1}=\mathbf{x}^{k}-J\left(\mathbf{x}^{k}\right)^{-1} \mathbf{F}\left(\mathbf{x}^{k}\right)
$$

where $\mathbf{x}$ is the unknown vector whose size is $N, \mathbf{F}(\mathbf{x})$ is the function vector, $J$ is the Jacobian matrix, and $k$ is the iteration index. The Jacobian matrix is given as

$$
J(\mathbf{x})=\left(\begin{array}{ccc}
\frac{\partial \mathbf{F}_{1}(\mathbf{x})}{\partial \mathbf{x}_{1}} & \cdots & \frac{\partial \mathbf{F}_{1}(\mathbf{x})}{\partial \mathbf{x}_{N}} \\
\vdots & \ddots & \vdots \\
\frac{\partial \mathbf{F}_{N}(\mathbf{x})}{\partial \mathbf{x}_{1}} & \cdots & \frac{\partial \mathbf{F}_{N}(\mathbf{x})}{\partial \mathbf{x}_{N}}
\end{array}\right)
$$

When $\mathbf{F}$ is not analytic function, the Jacobian matrix should be numerically evaluated. This numerical evaluation of the Jacobian matrix takes a lot of computation time. Since the computation of the inverse of the Jacobian matrix is computationally too expensive for a large size of the matrix, linear algebraic equations are constructed.

$$
J\left(\mathbf{x}^{k}\right)\left(\mathbf{x}^{k+1}-\mathbf{x}^{k}\right)=-\mathbf{F}\left(\mathbf{x}^{k}\right)
$$

Equation (3) can be solved by any fast linear algebraic equation solvers.

For the VLM inverse design, the chord and twist angle distributions are assigned as the unknown vector $\mathbf{x}$. If the number of the blade stations is equal to $N S$, the number of the unknowns becomes $N=2 N S$. F function is defined as the difference between the desired values and the computed values for the lift coefficient and axial induction. The computed lift coefficient and axial induction are provided by the VLM routine. For the Jacobian matrix, the second-order central finite

\footnotetext{
*Mechanical Engineer; Seongkyu.Lee@ge.com
} 
difference is used to find each element of the matrix in the current paper, which requires two computations of the VLM routine for each element. Two runs of the VLM routine provide $\partial \mathbf{F}_{1} / \partial \mathbf{x}_{i}$, $\partial \mathbf{F}_{2} / \partial \mathbf{x}_{i}, \ldots, \partial \mathbf{F}_{N} / \partial \mathbf{x}_{i}$ for each $i$ index, since the lift coefficient and axial induction distributions on the entire blade stations $(j=1,2, \ldots, N)$ are found from each VLM run. To complete the Jacobian matrix, the index $i$ needs to run from 1 to $N$. Therefore, the total number of the VLM run to construct the Jacobian matrix is equal to two times the number of the unknowns, i.e., $2 N$. For example, for 40 blade stations, the number of the unknowns is 80 , and the number of the VLM computation to construct the Jacobian matrix becomes 160 .

Figure 3 shows a flow chart of the VLM inverse design. At start, the initial blade geometry including the chord and twist angle distributions, the designed lift coefficient $\left(\mathrm{Cl}_{d}\right)$ and the axial induction $\left(a_{d}\right)$ distributions, and the operating conditions (TSR and Pitch angle) are read in. The chord and twist angle distributions are assigned as the unknown vector $\mathbf{x}$. Now the VLM code is run to provide $\mathbf{F}$ function and the Jacobian matrix. Again, this requires $2 \times N+1$ computations of the entire VLM routine for the Jacobian matrix and $\mathbf{F}$. Once the Jacobian matrix and $\mathbf{F}$ are obtained, the linear algebraic equation is solved to obtain new values of $\mathbf{x}$. At this step, the convergence check of $\mathbf{F}$ is performed. If $\mathbf{F}$ is not converged to zero, the new values of $\mathbf{x}$ are converted to the input to the VLM, and then the iteration is continued until F is converged to zero. When the convergence is met, the results are printed and the program is stopped.

\section{Numerical Results}

\section{Validation : Ideal Blade}

The inverse design code named VLMID is validated for a straight ideal blade in this section. The number of the blade staion is set to be 40 . Figure 4 shows a flow chart of the validation procedure for an ideal blade. First, the desired lift coefficient $\left(C_{l}\right)$ and axial induction $(a)$ are given to PROPID to calculate the chord and twist distributions. These determined chord and twist distributions are used as inputs to the VLM analysis code to provide computed $C_{l}$ and $a$. These computed $C_{l}$ and $a$ may not completely match with the initially desired $C_{l}$ and $a$, which are the inputs to PROPID, because of the code-to-code variation and 3-D effects which are more realistically accounted for by the VLM. The computed $C_{l}$ and $a$ are, in turn, used as new desired $C_{l}$ and $a$ for the VLMID. Given the new desired values, the chord and twist distributions are obtained by the VLMID. These chord and twist distributions are compared with the inputs of the VLM analysis for the validation of the inverse design. The desired $C_{l}$ and $a$ are assigned as the maximum lift coefficient and $1 / 3$ as described by the Betz limit [5], respectively. For this test, a GE18-13llr airfoil is used for the entire blade from the root to the tip. The polar data of this airfoil are the GE proprietary data and not shown in detail in the current paper.

Figure 5 (a) shows an outline of the Jacobian matrix. The row describes $f$ function, or the combination of the lift coefficient $\left(C_{l}\right)$ and axial induction $(a)$. The column indicates $x$ variables, or the combination of the twist $(t)$ and chord $(c)$. The first quadrant describes the rate of the change of the lift coefficient $\left(C_{l}\right)$ to the change of the twist $(t)$. The second quadrant represents the rate of the change of the lift coefficient $\left(C_{l}\right)$ to the change of the chord $(c)$. The third quadrant the rate of the change of the axial induction $(a)$ to the change of the twist $(t)$. The fourth quadrant the rate of the 


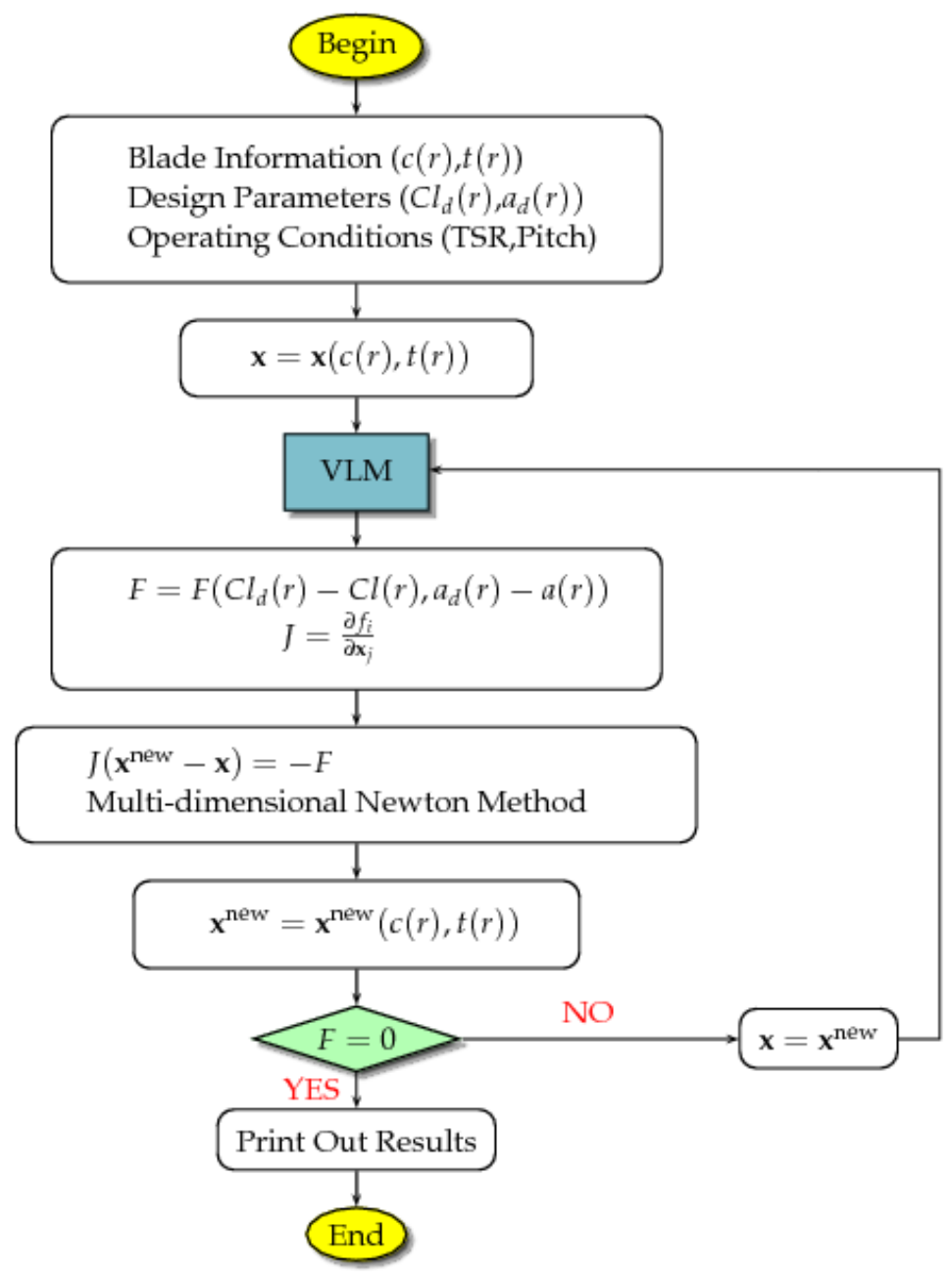

Figure 3: Flow chart of the inverse design of the VLM. 


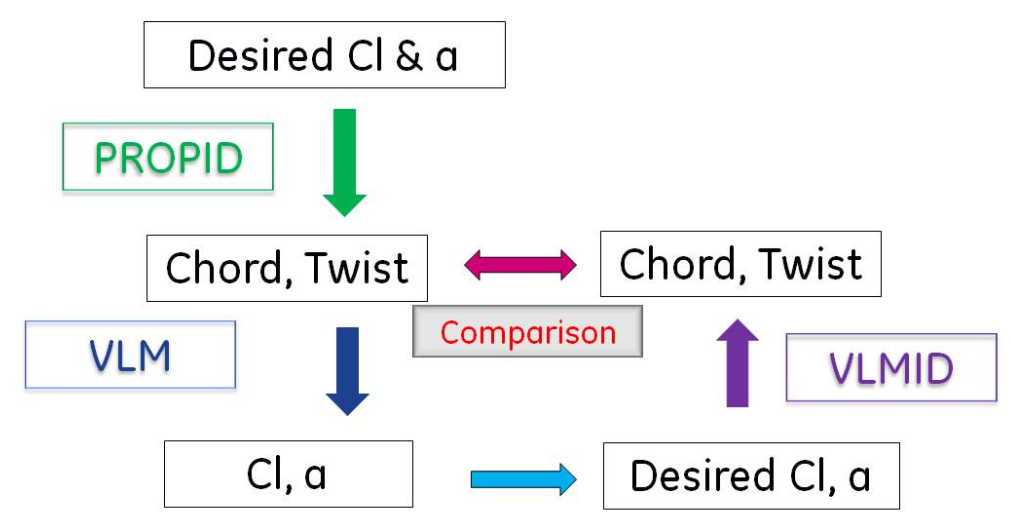

Figure 4: Schematic of the validation procedure for an ideal blade.

change of the axial induction (a) to the change of the chord (c). Figure 5 (b)shows the visualization of the Jacobin matrix for the ideal blade. The red color indicates the positive influence and blue negative influence. It is shown that the change of $t$ and $c$ influences $C_{l}$ and $a$ at not only the the blade station but also adjacent station positions, which accounts for 3-D effects. It is interesting to see a large influence of off-diagonal terms at the second quadrant. This indicates that the change of the chord at the outboard influences the lift coefficient at the inboard. This is thought to be the wake effect through the induced velocities.

Figure 6 shows the twist angle and $C_{l}$ distributions as a function of the normalized radial distance. In Fig. 6 (a), the input of the twist to the VLM analysis or the output of PROPID is defined as the correct twist, which is used to validate the output of the VLMID. In Fig. 6 (b), the desired $C_{l}$ is the output of the VLM analysis when the correct twist is used. This is also the input to the VLMID as the desired $C_{l}$. The desired $C_{l}$ is uniform in the middle section of the blade along the span with a value of 1.32. Rectangular symbols in Fig. 6 (a) represent the initial guess of the twist in the VLMID. A simple linear function with an arbitrary slope is used as the initial distribution of the twist. This linear twist distribution provides as small values of $C_{l}$ as 0.6 in the middle of the blade. Circular symbols in Fig. 6 represent the converged twist or the final output of the VLMID. It was found that the converged twist agrees very well with the correct one. Also, the converged $C_{l}$ matches very well with the desired one.

Figure 7 shows the normalized chord and axial induction as a function of the normalized radial distance. Again, a linear distribution was used as the initial guess of the chord for the VLMID. The desired induction is about -0.33 in the middle of the blade. It can be seen that the converged chord agrees very well with the correct one except a few points at the end of the root. The agreement between the desired and converged inductions is also excellent.

For this validation test, the convergence criteria of $1 \mathrm{e}-3$ was used. It was found that the solution was converged after 8 iterations of the Newton method, and the total computation time on a personal computer with $3.2 \mathrm{GHz}$ is around $5 \mathrm{~min}$. Given the accuracy of the solution and computation time, the inverse design for an ideal straight blade is considered to work very well. 


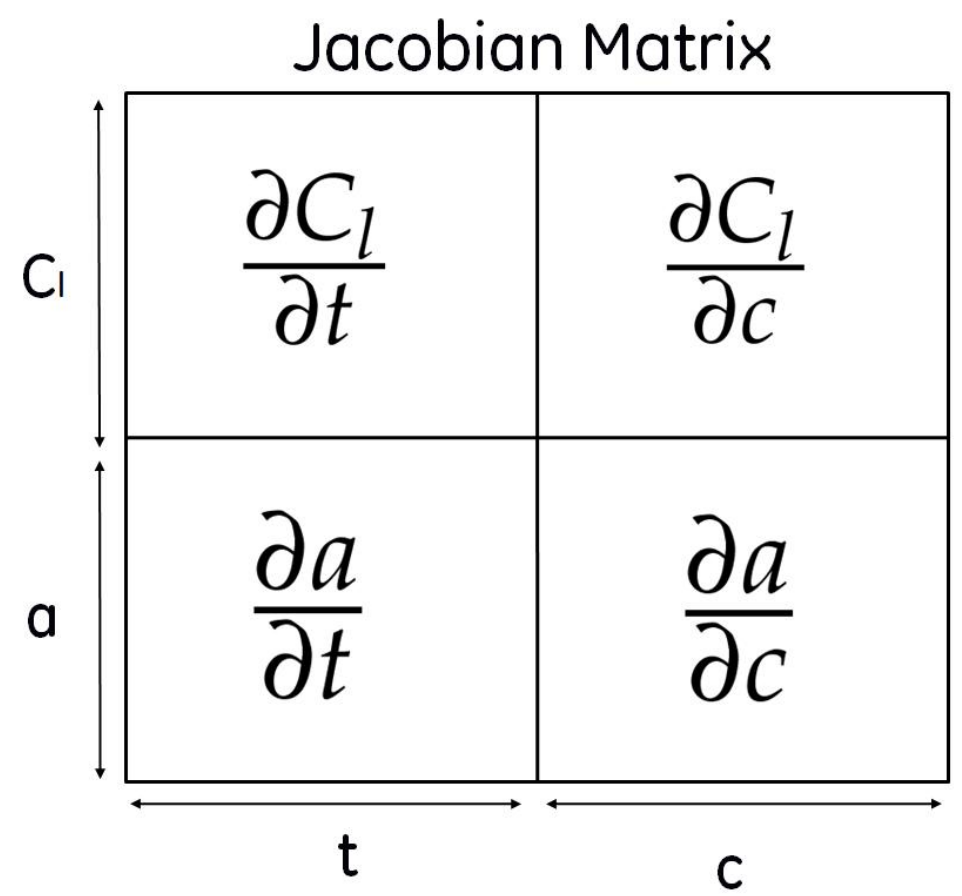

(a)

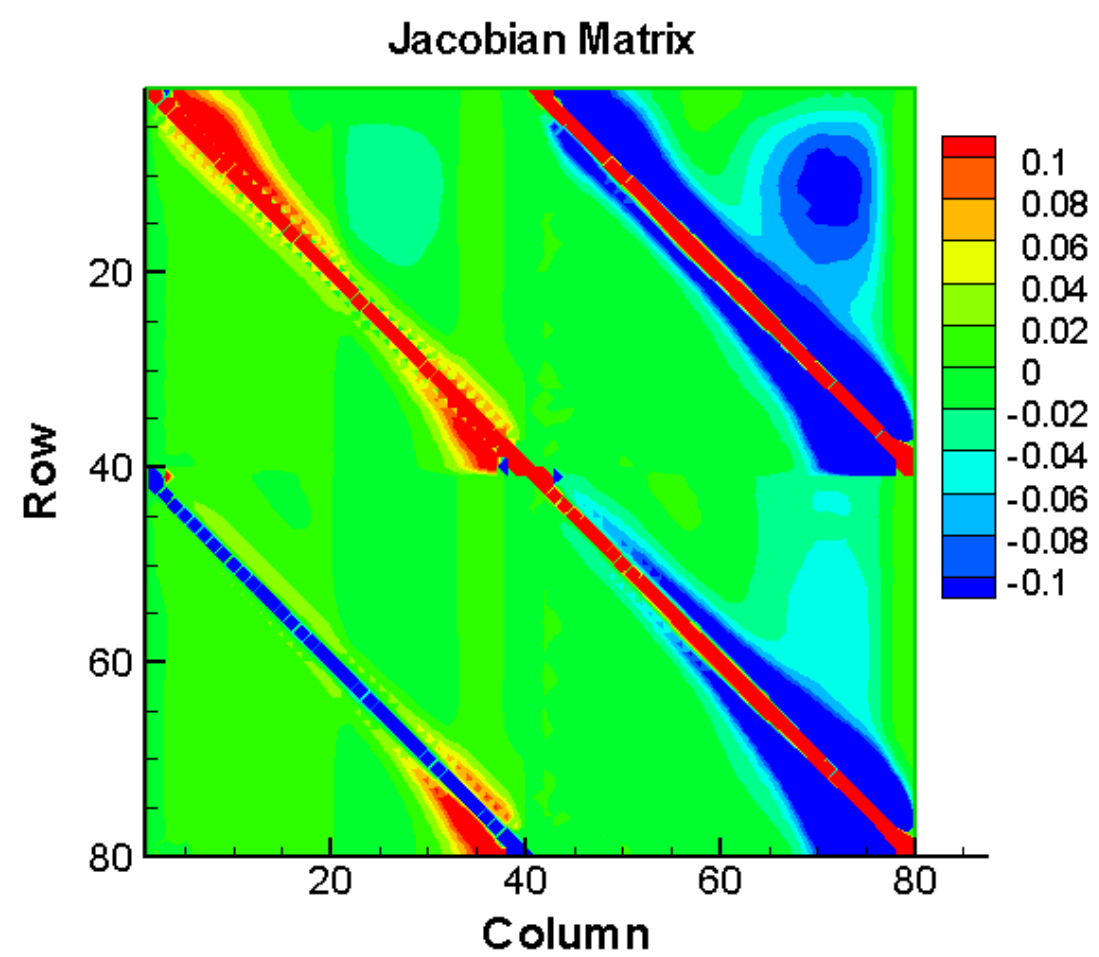

(b)

Figure 5: Visualization of the Jacobian matrix for an ideal blade 


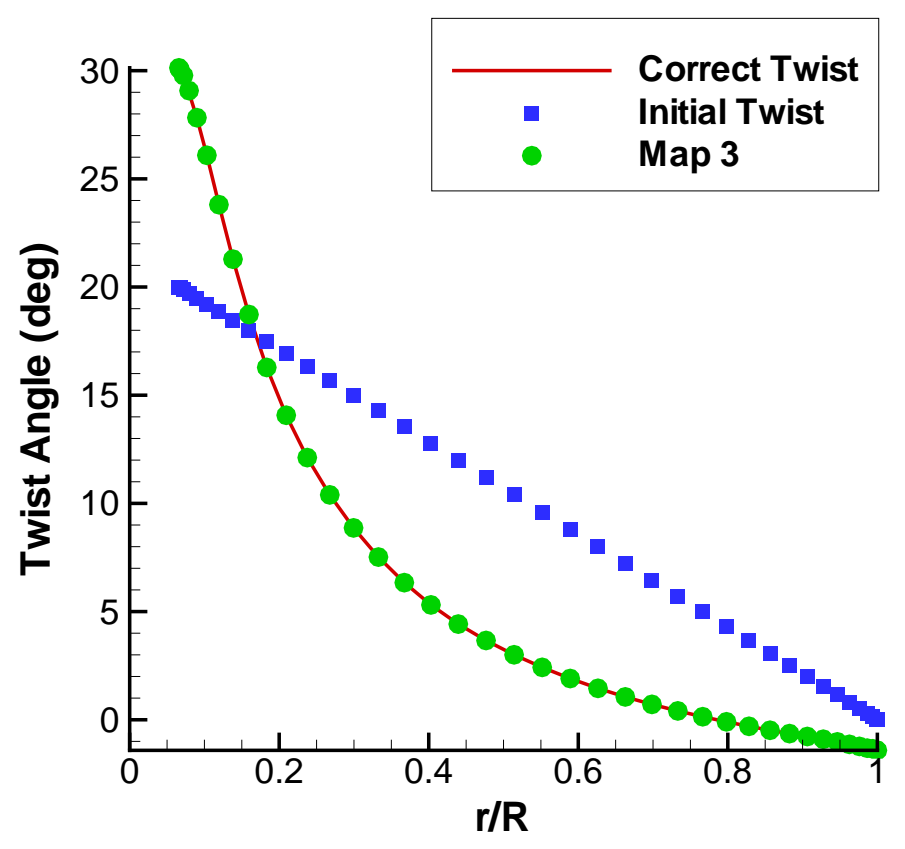

(a)

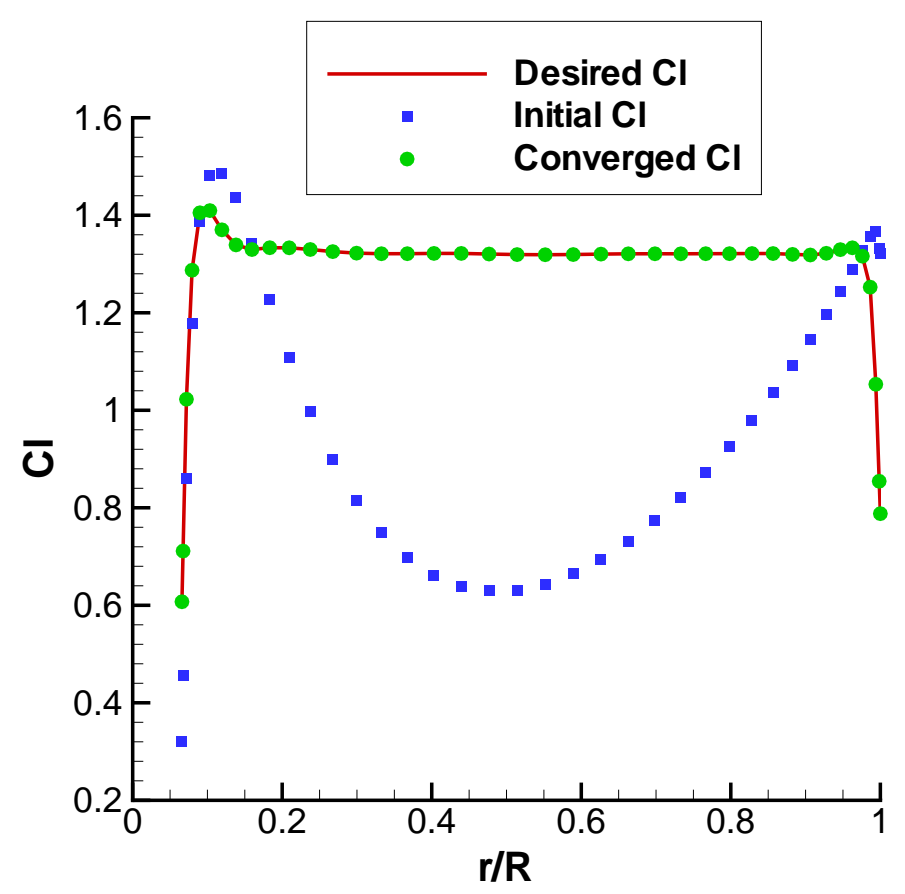

(b)

Figure 6: (a) Twist angle distribution, (b) $C_{l}$ distribution for a straight blade for the code validation. 


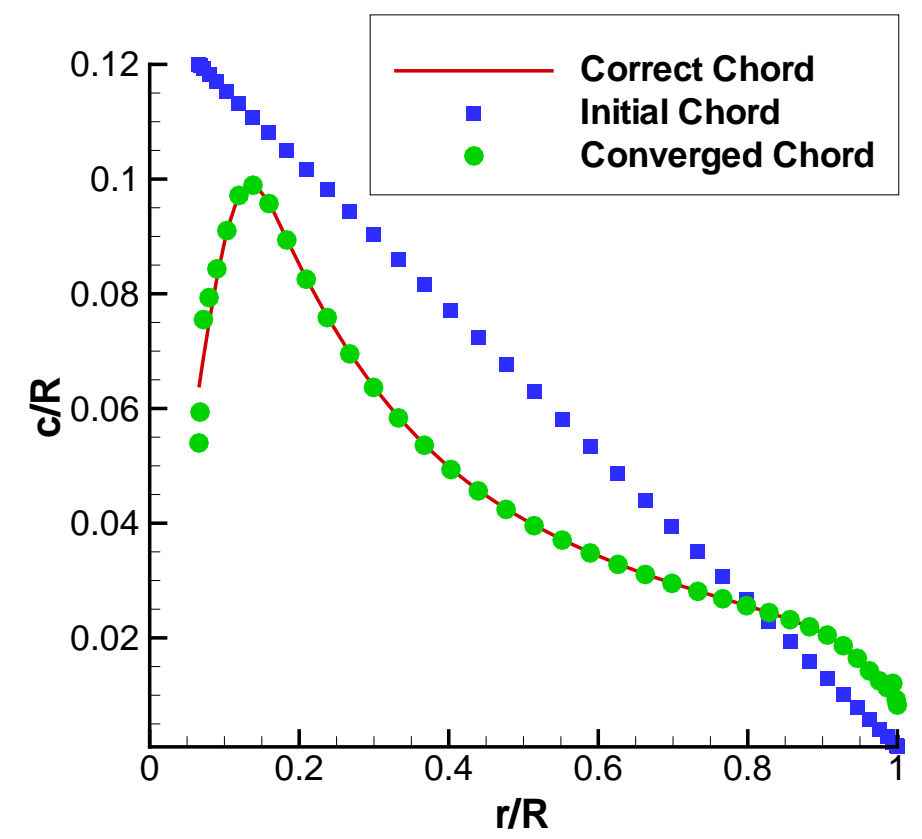

(a)

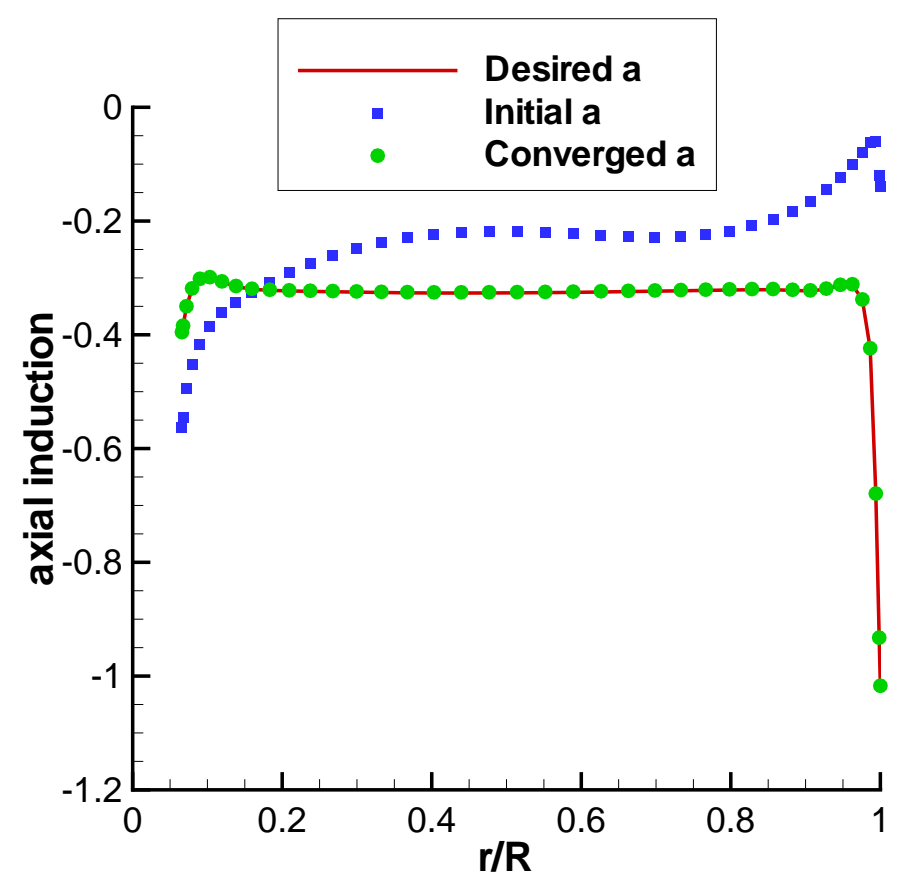

(b)

Figure 7: (a) Chord distribution, (b) axial induction distribution for a straight blade for the code validation. 


\section{Validation : NREL Blade}

In order to validate the code, a NREL wind turbine was tested. It is a 3 blade rotor with a $35 \mathrm{~m}$ blade radius. It is a straight blade with neither dihedra nor sweep angles. The tip speed ratio (TSR) was selected to be 6.25 . The wind speed was chosen to be $11.8 \mathrm{~m} / \mathrm{s}$. 40 radial stations were selected on the blade for the VLMID computation. Figure 8 shows twist and $C_{l}$ distributions as a function of normalized radial location. For the code validation, the $C_{l}$ distribution obtained from the VLM analysis with the correct twist distribution (solid line in (a)) was used as the desired $C_{l}$ distribution (solid line in (b)). If the code succeeds, the converged twist and $C_{l}$ distributions should match the correct and desired distributions, respectively. Initially, a simple linear function was used for the twist distribution (rectangular symbols in (a)). This results in very different $\mathrm{C}_{l}$ distribution (rectangular symbols in (b)) from the desired values. The convergence criteria of $1 e-2$ is used for the $L 2$ norm of the twist angle. It converges within 10 iterations and the computation time is within $5 \mathrm{~min}$. The converged twist and $C_{l}$ distributions are shown in Fig. 8 as circular symbols. Note that the twist angle has a very large oscillation around $r / R=0.2$. Also, the hub and tip points have very large deviations of the twist angle from adjacent values. However, the converged $C_{l}$ agrees very well with the desired $C_{l}$. In order to remove the high frequency oscillations and abnormal behavior at the hub and tip, a low-pass filter and extrapolation for the hub and tip from the adjacent points were used at each Newton iteration. The results are shown as triangular symbols. It can be seen that the filtering and extrapolation provides excellent agreement between the converged and correct twist distributions and between the converged and correct $C_{l}$ distributions.

\section{References}

[1] J. M. Hallissy and J. J. Chattot. Validation of a helicoidal vortex model with the nrel unsteady aerodynamic experiment. Computational Fluid Dynamics Journal, 14(3), 2005.

[2] J. J. Chattot. Helicoidal vortex model for steady and unsteady flows. Computers and Fluids, 35(7), 2006.

[3] J. J. Chattot. Tower shadow modelization with helicoidal vortex method. Computers and Fluids, 37(5), 2008.

[4] J. J. Chattot. Effect of blade tip modifications on wind turbine performance using vortex model. Computers and Fluids, 38(7), 2009.

[5] J. Gordon Leishman. Principles of Helicopter Aerodynamics,. Cambridge University Press, New York, 2000. 


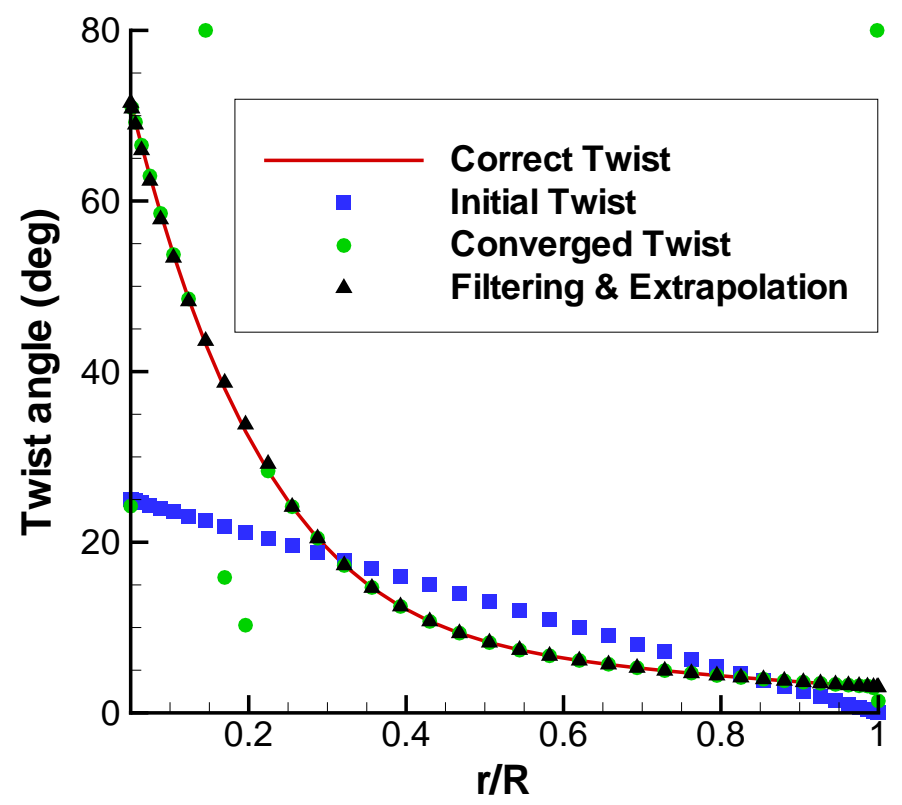

(a)

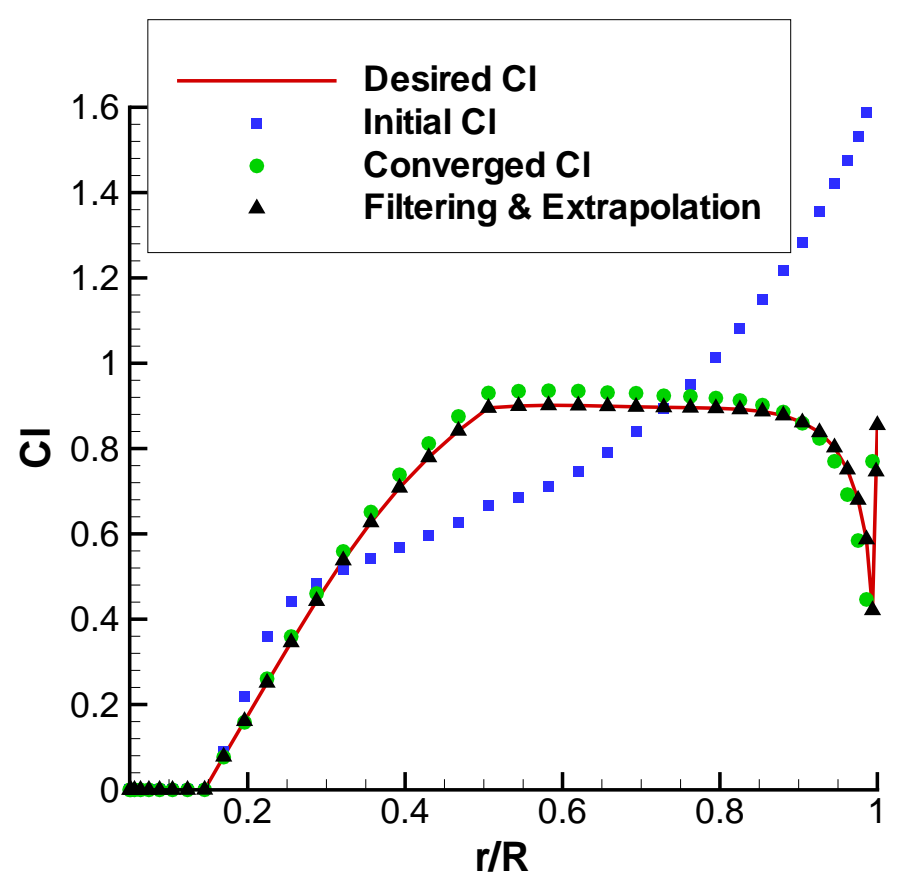

(b)

Figure 8: (a) Twist angle distribution, (b) $C_{l}$ distribution for a NREL wind turbine for the code validation. 


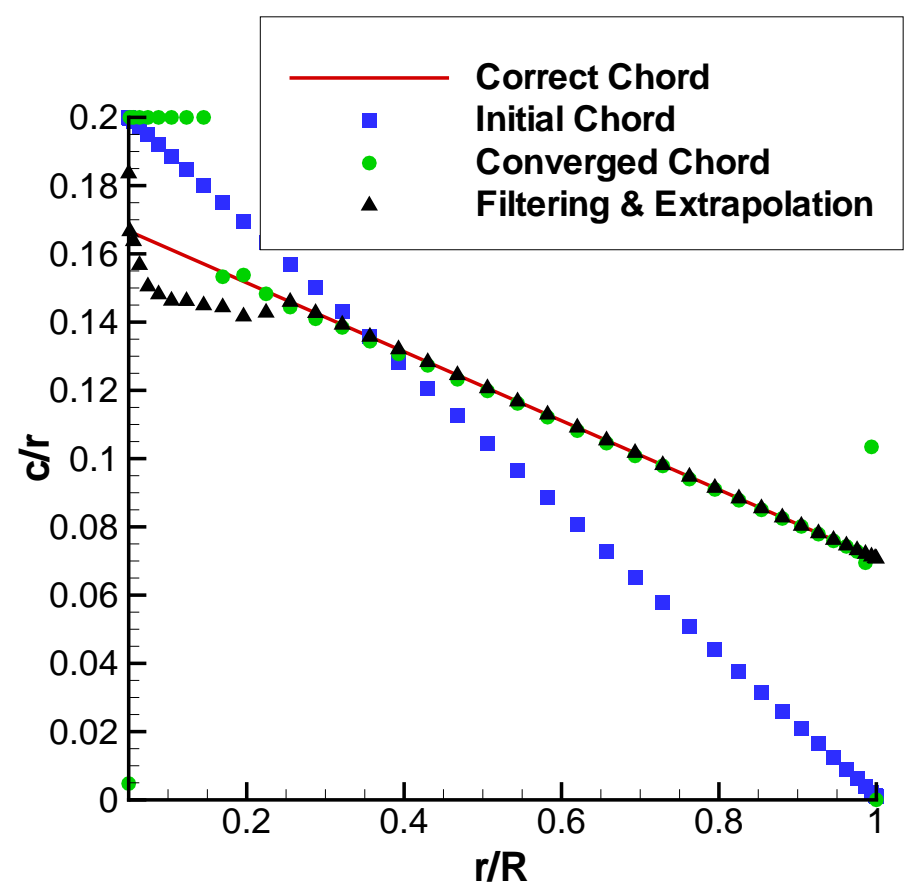

(a)

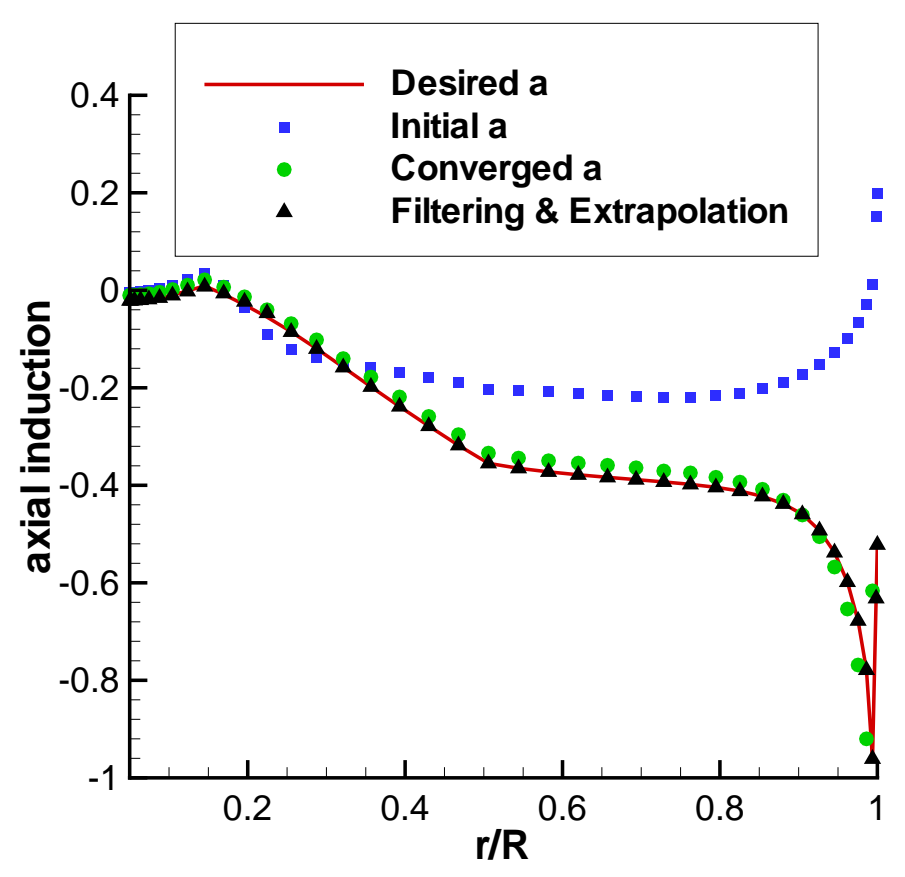

(b)

Figure 9: (a) Chord distribution, (b) axial induction distribution for a NREL wind turbine for the code validation. 


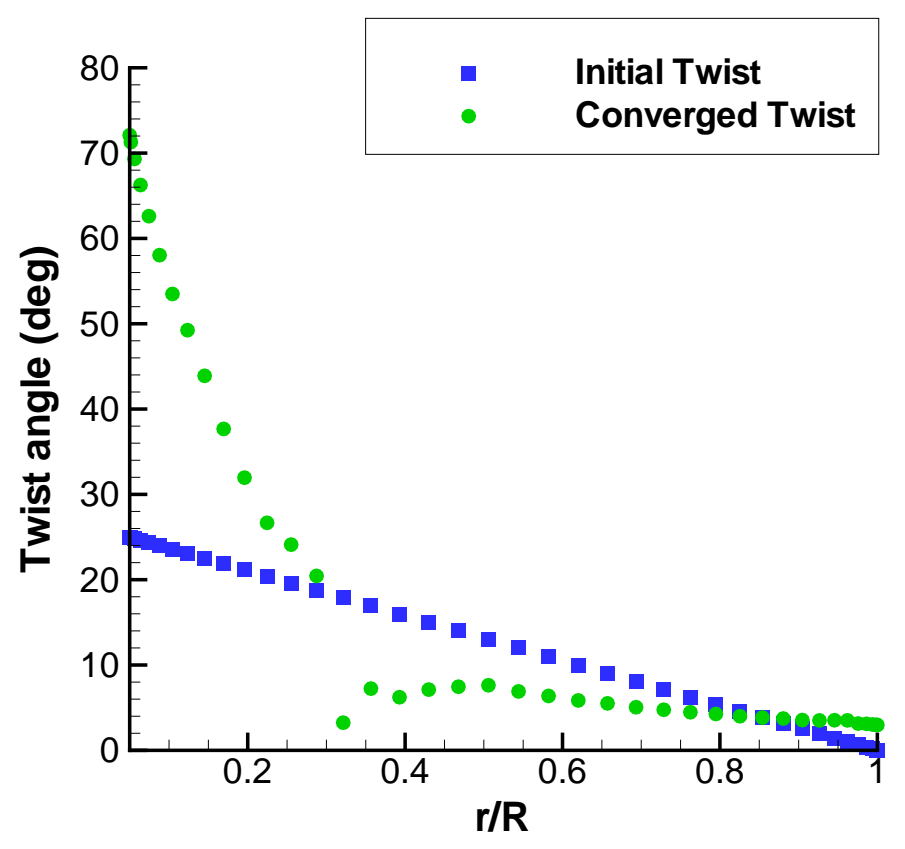

(a)

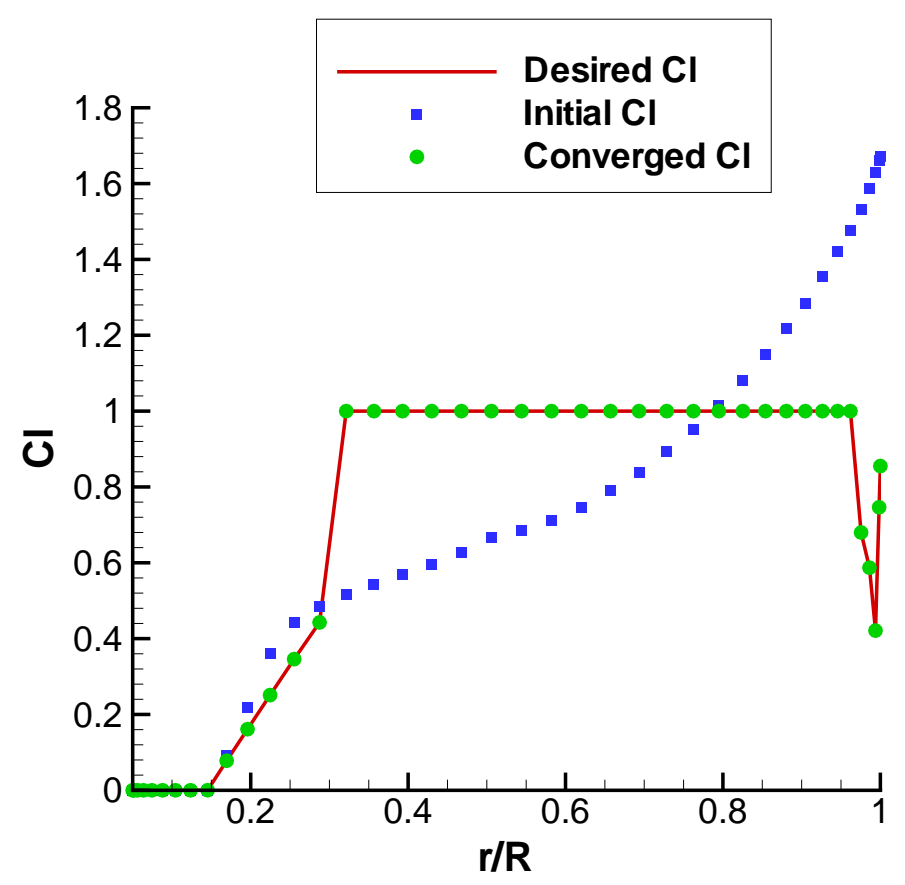

(b)

Figure 10: (a) Twist angle distribution, (b) $\mathrm{Cl}$ distribution for a new NREL wind turbine. 


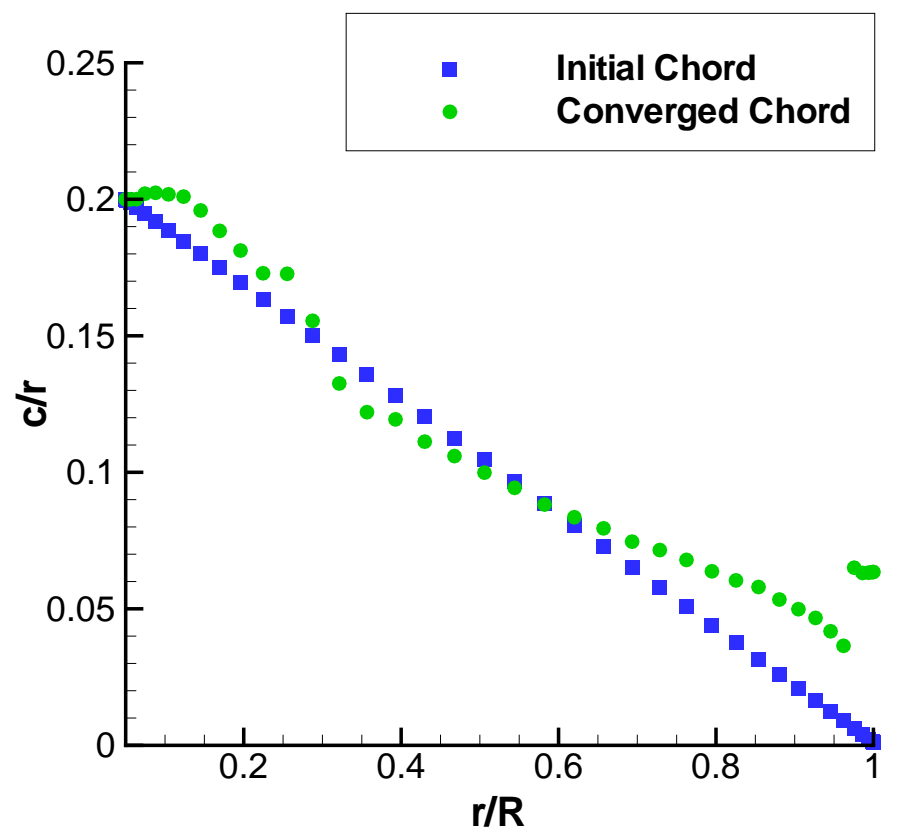

(a)

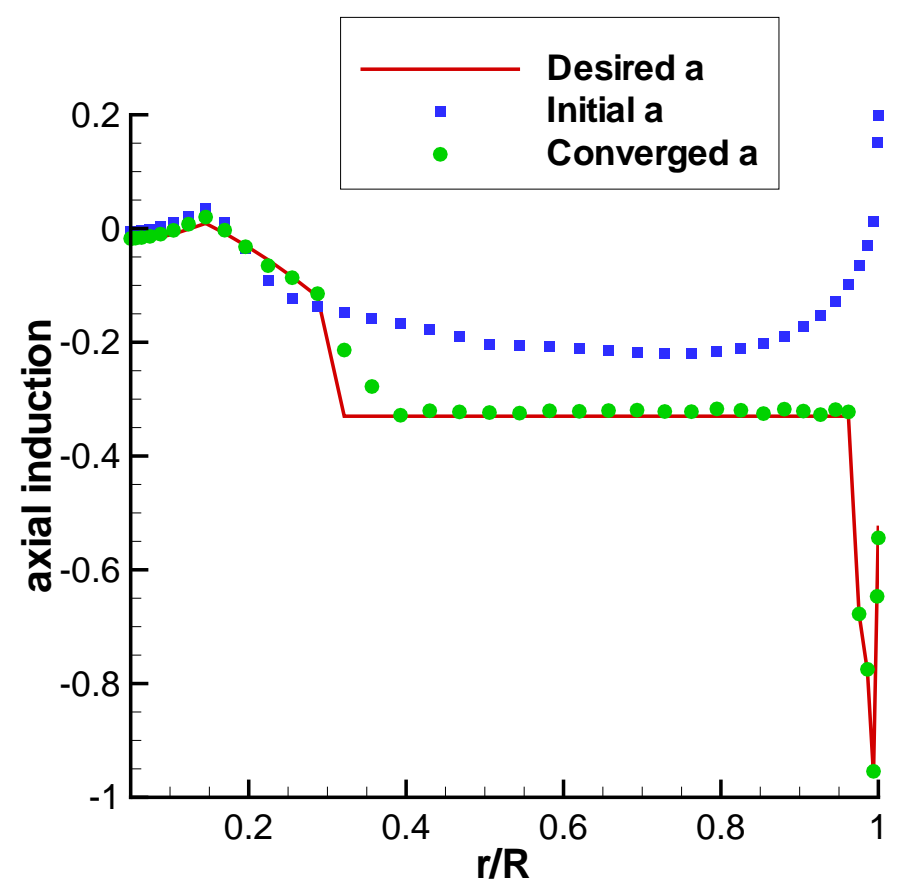

(b)

Figure 11: (a) Chord distribution, (b) axial induction distribution for a new NREL wind turbine. 